\title{
Swiss Quality Award: die Gewinner 2011
}

\author{
Über 100 Projekte wurden für den diesjährigen Swiss Quality Award eingereicht. An- \\ lässlich des vierten Nationalen Symposiums für Qualitätsmanagement im Gesund- \\ heitswesen hat die Jury nun vier herausragende Projekte ausgezeichnet. Die Preis- \\ verleihung des mit viermal 10000 Franken dotierten Wettbewerbs fand am 12. Mai \\ 2011 in Bern statt.
}

Jürg Beutler ${ }^{a}$,

Matthias Scholer ${ }^{b}$

a Kommunikation FMH

b Wissenschaftsjournalist, vetcom.ch

Korrespondenz:

FMH

Varja Nikolic

Daten, Demographie und

Qualität DDQ

Elfenstrasse 18

Postfach 170

CH-3000 Bern 15

ddq@fmh.ch

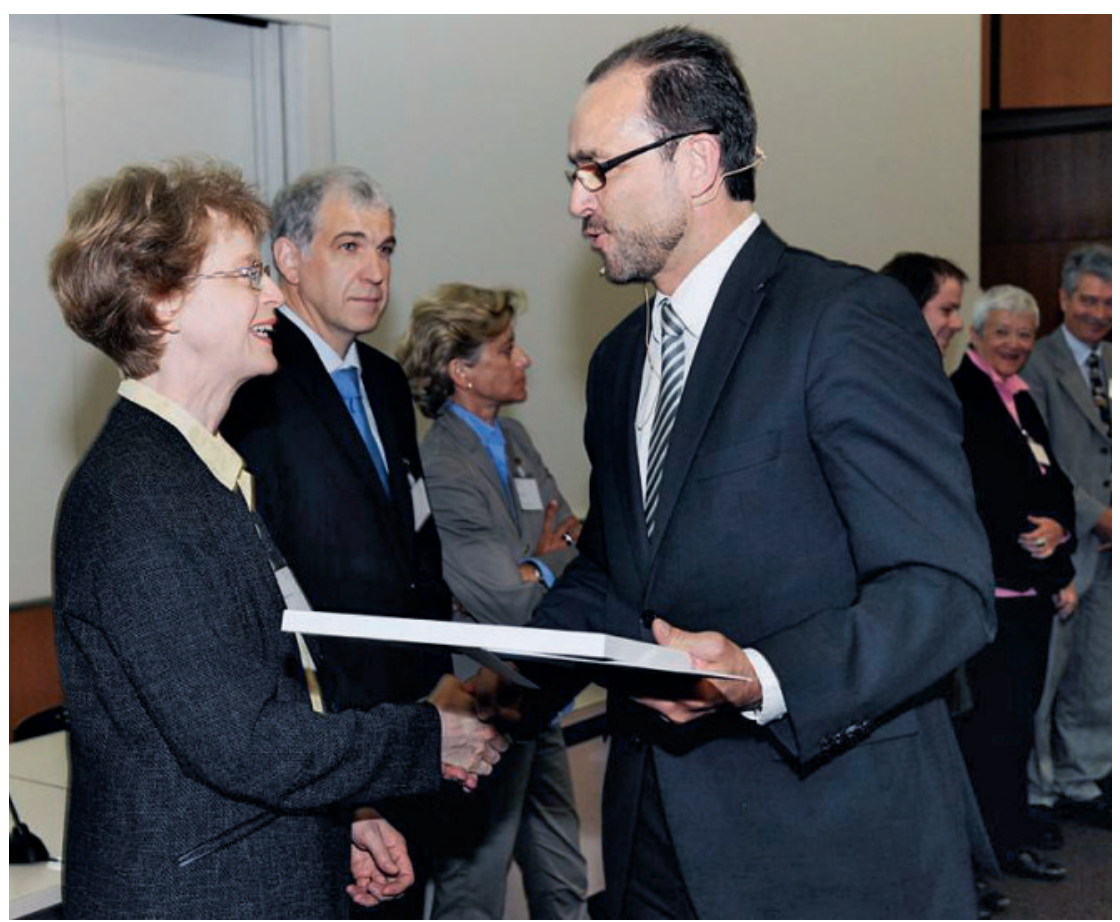

Preisübergabe durch Pascal Strupler, Direktor BAG.
Qualität fällt nicht vom Himmel, Qualität bedeutet harte Arbeit und grossen Einsatz. Der Swiss Quality Award steht für dieses Engagement. Er prämiert herausragende Innovationen im Gesundheitswesen, die im Qualitätsmanagement neue Impulse setzen. Wie weit und wie vielfältig dieses Engagement in der Schweiz reicht, hat die vierte Verleihung des Swiss Quality Awards 2011 gezeigt: Über 100 vielversprechende Projekte gingen in den vier Preiskategorien Management, Patientensicherheit, Technologie und Information ein - davon

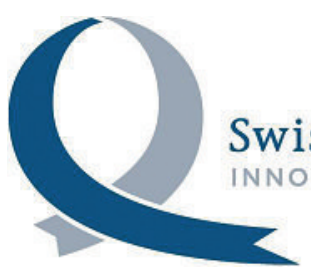
Rahmen des vierten Symposiums für Qualitätsmanagement im Gesundheitswesen mit einem Projektposter vorgestellt. wurden mehr als $30 \mathrm{im}$

\section{Grosse Bandbreite}

Was sofort auffällt: Die eingereichten Innovationen decken die unterschiedlichsten Bereiche der medizinischen Qualitätsarbeit ab. Das Spektrum reicht von technischen Innovationen, praktischen Patientenbroschüren mit Handlungsanweisungen bis zu Datenpools für evidenzbasierte Empfehlungen oder Ratgeber-Websites für Patienten. Die grosse Bandbreite spiegelt sich auch in den Teams, die hinter den Innovationen stehen: Arztpraxen sind ebenso vertreten wie Universi-

tätskliniken, Unternehmungen oder Gesundheitsorganisationen. Der gemeinsame Nenner dabei: Alle Projekte liefern Antworten auf konkrete Probleme und haben sich in der Praxis bereits bewährt - mit echtem Nutzen für die Patientinnen und Patienten.

Für die Bewertung der verschiedenen Innovationen verpflichteten die Veranstalter des Swiss Quality Awards eine Fachjury, der auch internationale Qualitätsexpertinnen und -experten angehörten. Sämtliche Projekte wurden für die Bewertung anonymisiert und von mehreren Jurorinnen und Juroren beurteilt. Dabei kam auch ein transparenter Kriterienkatalog zum Einsatz. So hat die Jury zum Beispiel den Innovationsgehalt, die Kundenorientierung oder die Nachhaltigkeit berücksichtigt.

\section{Die vier Gewinnerprojekte}

Vier Qualitätsinnovationen hat die Jury als besonders herausragend bewertet und einstimmig mit dem Swiss Quality Award 2011 prämiert. Hinter jedem der vier Gewinnerprojekte stehen engagierte Teams, welche die Projekte von der Anfangsidee bis zur finalen Umsetzung begleitet haben. Professor Philipp Schneider, Präsident der Schweizerischen Gesellschaft für Qualitätsmanagement im Gesundheitswesen und Mitveranstalter des Symposiums, würdigte sie als engagierte Mitarbeitende, die lösungsorientierte Projekte ohne viel Aufsehen entwickelt und grossartige Arbeit geleistet haben. Wie zukunftsweisend und 

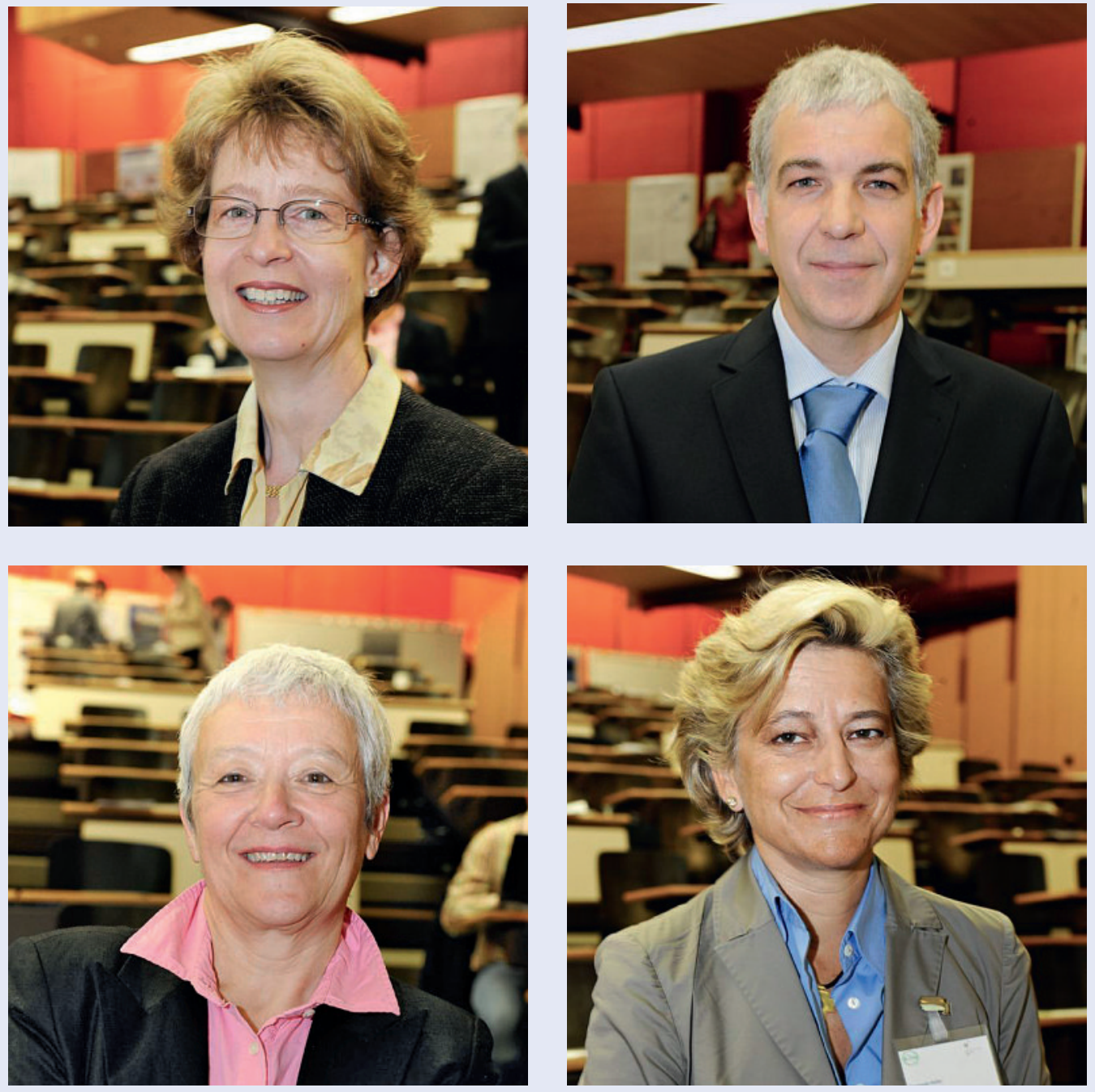

Stellvertretend für ihre Teams stellten sie an der Preisverleihung die Gewinnerprojekte vor (im Uhrzeigersinn): Kategorie «Management»: Eva Maria Genewein Depelteau; Kategorie «Patientensicherheit»: Martin Egger; Kategorie «Technologie»: Emanuela Keller; Kategorie «Information»: Margareta Schmid.

erfolgversprechend die preisgekrönten Qualitätsinnovationen sind, zeigte sich, als die Projektleiter ihre Projekte - stellvertretend für die gesamten Teams vorstellten.

\section{Kategorie «Management»}

Overflow-Management: Bewältigung von Spitzenzeiten und Reduktion der Kapazitätsengpässe im Notfallzentrum von 60 auf 10/Jahr im UNZ

In der Vergangenheit musste das Universitäre Notfallzentrum des Inselspitals Bern (UNZ) wegen massiver Überbelastung mehrfach schliessen. Für alle Betroffenen eine unbefriedigende Situation. Einer aus Ärzten und Pflegenden bestehenden Arbeitsgruppe gelang es, mit der Entwicklung und Einführung geeigneter
Massnahmen die Häufigkeit solcher Kapazitätsengpässe signifikant zu senken. Die dabei gewonnenen Erkenntnisse lassen sich auch auf andere Notfallzentren bzw. Abteilungen übertragen.

\section{Kategorie «Patientensicherheit»}

Reduktion von Urinkathetertagen und Antibiotika-Behandlungstagen für Harnwegsinfektionen in der stationären Akutmedizin Eine wissenschaftliche Untersuchung des Regionalspitals Emmental und des Instituts für Infektionskrankheiten der Universität Bern zeigt: Durch einen bewussten Verzicht auf überflüssige Interventionen kann in gewissen Bereichen mehr für die Patientensicherheit getan werden als durch teure technische Innovationen. 


\section{Kategorie «Technologie»}

Optical technologies for combined monitoring of cerebral blood flow and oxygen metabolism to improve outcome in patients with stroke, traumatic brain injury and after cardiac arrest Ein Forscherteam der ETH Zürich und des Universitätsspitals Zürich hat zwei Methoden entwickelt, mit denen sich die Sauerstoffversorgung geschädigter Hirnregionen äusserst genau messen lässt. Diese Innovationen haben das Potential, die Gefahr von Folgeschäden nach einem Schlaganfall oder einem Herzstillstand massgeblich zu mindern.

\section{Kategorie «Information»}

\section{Gesundheitscoaching - Patient und Arzt als Partner}

Entscheiden sich Patienten, der Gesundheit zuliebe ihren Lebensstil zu ändern, können sie dabei auf ein massgeschneidertes Coaching seitens ihrer Hausärztinnen oder Hausärzte zählen. So lässt sich das Ziel eines neuen Programmes des Kollegiums für Hausarztmedizin zur Erhöhung der Gesundheitsförderung und Prävention zusammenfassen. Die ersten Erfahrungen nach einer Pilotphase im Kanton St.Gallen sind äusserst vielversprechend!

\section{Grosse Ehre für die Preisträger}

Die vier Gewinnerteams erhalten nicht nur ein Preisgeld von je 10000 Franken, sie dürfen sich ab sofort «Gewinner des Swiss Quality Awards 2011» nennen.
Pascal Strupler, Direktor des Bundesamtes für Gesundheit BAG, zeigte sich bei der Preisübergabe beeindruckt von den Projekten: «Solche Arbeiten zeigen uns, dass das Qualitätsmanagement im Gesundheitswesen keineswegs in der Sackgasse steckt - im Gegenteil: Sie sind für alle Akteure im Gesundheitswesen Ansporn, sich kontinuierlich weiterzuentwickeln.»

Hinter dem Swiss Quality Award steht die Verbindung der Schweizer Ärztinnen und Ärzte FMH, das Institut für Evaluative Forschung in der Medizin IEFM der Universität Bern und die Schweizerische Gesellschaft für Qualitätsmanagement im Gesundheitswesen SQMH. «Patientinnen und Patienten sind auf Fortschritte im Qualitätsmanagement angewiesen. Der Swiss Qualität würdigt Projekt, die sich für dieses Ziel einsetzen», sagt FMH-Zentralvorstandsmitglied Daniel Herren zum Engagement der FMH.

\section{Mehr erfahren}

Der Swiss Quality Award ist mehr als eine Auszeichnung: Alle am Nationalen Symposium für Qualitätsmanagement im Gesundheitswesen vom 12. Mai 2011 präsentierten Projekte werden auch auf www.swissqualityaward.ch $(\rightarrow$ Die Projekte) vorgestellt, damit sich Fachpublikum und Öffentlichkeit darüber informieren können. Es steht eine praktische Suchmaschine zur Verfügung.

Eine Zusammenfassung der vier Siegerprojekte erscheint in den beiden nächsten Ausgaben der Schweizerischen Ärztezeitung. 